\title{
LA SOCIEDAD CIENTÍFICA DE ESTUDIANTES DE MEDICINA HUMANA DE LA UNIVERSIDAD RICARDO PALMA: ESTUDIANTES MÁS ALLÁ DE LAS AULAS
}

\author{
THE SCIENTIFIC SOCIETY OF STUDENTS OF HUMAN MEDICINE ATTHE UNIVERSITY RICARDO PALMA : \\ STUDENTS BEYOND THE CLASSROOM \\ Mariela Vargas', Katerine M. García-Moreno', Matlin M. Cárdenas
}

\begin{abstract}
a Universidad está definida como el espacio donde se proporciona conocimiento superior y especializado para cada rama del saber; este hecho concentra el desempeño tanto del maestro como del estudiante, pues ambas partes deben estar dispuestas y comprometidas con la realización de nuevos estudios, que se conviertan en un aporte para la sociedad.
\end{abstract}

Es por ello, que la Facultad de Medicina de la Universidad Ricardo Palma (FAMURP) promueve en sus estudiantes y docentes la educación de calidad, la investigación y la creación de una cultura humanística. Dentro de este contexto, nace la Sociedad Científica de Estudiantes de Medicina Humana de la Universidad Ricardo Palma (SOCEMURP) que desde su fundación el 6 de julio del 2005, ha fomentado la producción científica de calidad, el desarrollo de actividades académicas y la extensión hacia la comunidad.

SOCEMURP cuenta con el apoyo y la supervisión del Instituto de Investigación en Ciencias Biomédicas (INICIB) de la FAMURP, quienes en trabajo conjunto buscan promover en los estudiantes de medicina la pasión por la investigación, la participación en actividades académicas y preventivas - promocionales hacia la comunidad.

A nivel nacional, SOCEMURP es miembro activo de la Sociedad Científica Médico Estudiantil Peruana (SOCIMEP) - ente que congrega a 37 sociedad científicas Sociedades Científicas de Estudiantes de Me- dicina distribuidas en 15 regiones del Perú- y a nivel internacional integra la Federación Internacional de Asociaciones de Estudiantes de Medicina (IFMSA) organización no gubernamental, que integra más de 127 organizaciones miembros de 119 países en seis continentes.

En sus más de 10 años de creación, SOCEMURP ha crecido en número de miembros y complejidad, distribuyendo sus funciones y actividades en sus diversos comités, siendo un ejemplo de organización, cooperación y trabajo en equipo.

\section{INVESTIGACIÓN, PILAR FUNDAMENTAL}

Desde sus inicios, SOCEMURP impulsó arduamente uno de sus principales pilares, la investigación; a través del Comité Permanente Científico (CPC-SCORE) encargados de brindar capacitaciones y talleres en el área, bajo la asesoría de docentes calificados, generando interés y oportunidades en la realización de casos clínicos, proyectos y trabajos de investigación; contribuyendo en la formación de futuros médicos investigadores. Fomentando una cultura de investigación, hecho demostrado, no sólo por las publicaciones realizadas, sino por la participación de los estudiantes, en eventos científicos nacionales e internacionales, quienes han logrado destacar en los primeros lugares, siendo un motivo de orgullo.

Es importante comprender que realizar una investigación es una labor que tiene como fin la publicación científica, en un espacio en el cual el co- 
nocimiento aportado se registre como un hecho verídico, es por eso que el Comité Permanente de Publicaciones Científicas (CPPC) promueve y asesora la publicación de los trabajos realizados por CPC-SCORE, y es en trabajo conjunto de ambos comités, que se han logrado el incremento exponencial del número de publicaciones realizadas en los últimos 5 años.

\section{PROMOVIENDO LA EDUCACIÓN DE CALIDAD}

El Comité Permanente Académico (CPA-SCOME) busca fortalecer el nivel académico de los estudiantes de la FAMURP, reforzando temas relevantes en investigación y en la práctica médica, en coordinación y asesoría de docentes calificados. Motivo por el cual, se han implementado ciclos de conferencias, charlas informativos, talleres con el único fin, de potencializar los conocimientos en los diversos temas de salud.

La participación de los estudiantes, busca incentivar la vocación hacia la docencia, fortaleciendo habilidades y conocimientos en las diversas áreas del saber médico.

\section{SOCEMURP, TRABAJANDO CON Y PARA LA COMUNIDAD}

El Comité Permanente de Atención Integral en Salud (CPAIS) junto a sus tres subcomités CPAIS-SCORP, CPAIS-SCORA y CPAIS-SCOPH desarrolla actividades preventivo-promocionales en trabajo conjunto con las comunidades. SOCEMURP ha sido partícipe de diversos proyectos locales y nacionales, como los Campamentos Multidisciplinarios de Investigación y Servicios (CUMIS) donde estudiantes de diversas facultades de Medicina fortalecieron sus capacidades de liderazgo, organización y trabajo multidisciplinario.

\section{INTERCAMBIOS CLÍNICOS Y DE INVESTIGACIÓN}

Desde su incorporación a IFMSA-Perú, los estudiantes de la FAMURP han sido participes de diferentes actividades de interculturalidad, accediendo a plazas de intercambio clínico en diferentes especialidades y países como Brasil, República Checa, México, Colombia, entre otros; de igual forma, SOCEMURP ha recibido estudiantes extranjeros de diversos continentes quienes han rotado en diversas especialidades clínicas y quirúrgicas.

Actualmente, gracias al apoyo de las autoridades de nuestra universidad, se logró la apertura de los Intercambios de investigación dentro de la FAMURP y se espera que en los próximos meses, se pueda recibir a los primeros intercambistas, quienes harán uso de los laboratorios, siendo participes de trabajos de investigación dirigidos por docentes y alumnos investigadores.

\section{PERSPECTIVAS FUTURAS}

El ser parte de la Sociedad Científica de Estudiantes de Medicina de la Universidad Ricardo Palma es una oportunidad para poner en acción inquietudes y desarrollar diferentes habilidades, adquirir experiencias que tienen un importante valor curricular para la Facultad de Medicina Humana y el Instituto de Investigación en Ciencias Biomédicas de la Universidad Ricardo Palma, los cuales realizan un continuo fortalecimiento de habilidades científicas, académicas y humanisticas, preparando a médicos íntegros, para desarrollarse en un mundo globalizado.

Fuente de financiamiento: Autofinanciado.

Conflicto de interés: Los autores declaran no tener conflictos de interés en la publicación de este artículo.

Recibido: 08/03/2016 / Aprobado: 29/04/2016 